\title{
InfraRed NDT for cured prepreg carbon specimens
}

\author{
by P. Bagavac*, L. Krstulović-Opara* and Ž. Domazet*
}

* Faculty of Electrical Engineering, Mechanical Engineering and Naval Architecture, University of Split, Croatia, (petra.bagavac; opara; domazet)@fesb.hr

\section{Abstract}

Prepreg laminated composite plates with Teflon inserts and sandwich panel with damaged Coremat were subjected to InfraRed Non-Destructive Testing. Every sample was recorded by means of reflected thermography and raw data were processed by various algorithms: Thermal Signal Reconstruction, Fast Fourier Transformation and Principal Component Analysis.

\section{Introduction}

Prepreg is a term for "pre-impregnated" composite fibers where a matrix material, such as epoxy, is already present. Prepreg allows one to impregnate the fibers on a flat workable surface, or rather in an industrial process, and then later form the impregnated fibers to a shape which could prove to be problematic for the common production processes, such as hot injection process. Recently, there has been considerable interest in prepregs and therefore for non-destructive testing of manufactured parts.

\section{Materials and methods}

Three prepreg carbon samples were tested, Fig. 1. Specimen CFRP-T001 is six-plies thick and damages were simulated as Teflon inserts with different geometries (shape and area) at the same depth. Specimen CFRP-T002 is sixplies thick and damages were simulated as Teflon inserts with same shape but different area at different depth. Specimen SENDWICH001 is sandwich composite material where Coremat was inserted between four-plies of carbon. Coremat was previously drilled with drills of different diameters. The specimens were made from RAW006-010319 SHD DF314-C650HS-3K-1250mm carbon prepregs and matured for 18 hours in autoclave at $75{ }^{\circ} \mathrm{C}$ and 1.5 bar pressure.

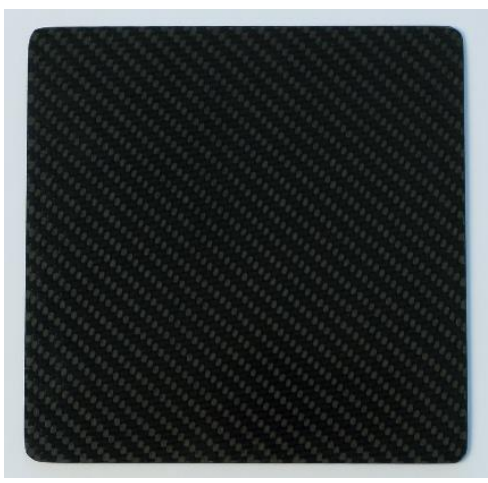

CFRP-T001

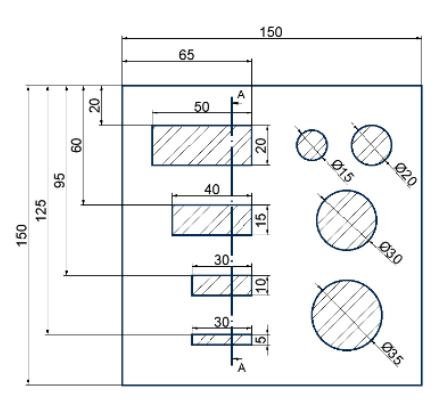

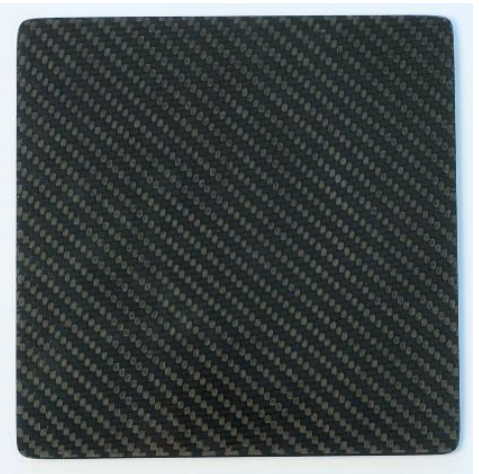

CFRP-T002

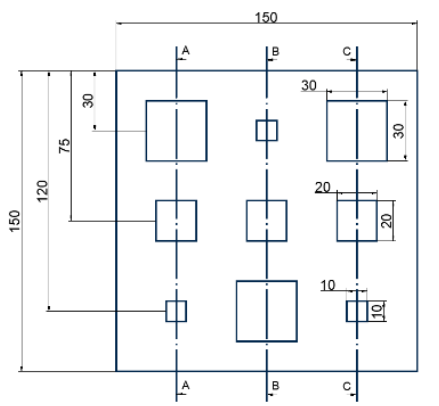

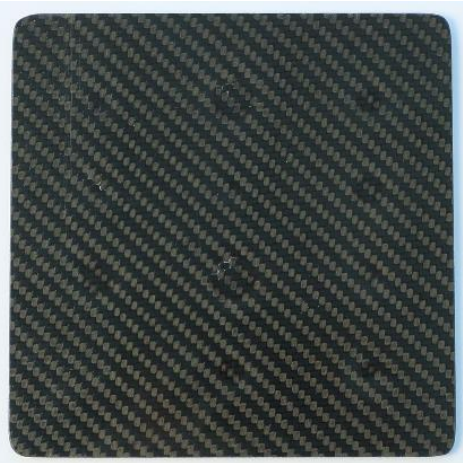

SENDWICH001

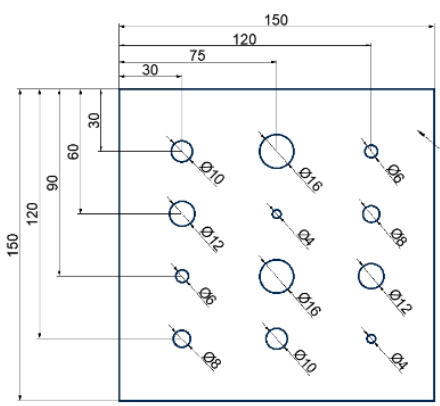

Fig. 1. Samples: a) CFRP-T001, b) CFRP-T002 and c) SENDWICHOO1 
Samples were tested with reflective thermography. Experimental setup included MW FLIR 5000SC infrared camera with acquisition frequency set to $25 \mathrm{fps}$ and photographic flash lamp TRIA PRO connected with HENSEL flash generator able to emit $6000 \mathrm{~J}$ in $1 / 440 \mathrm{~s}$. Raw data were processed in Matlab with TSR ( $1^{\text {st }}$ and $2^{\text {nd }}$ derivation), PPT and PCA [1-3].

\section{Results and conclusion}

Damage is poorly seen on raw data images, as expected, since numerous influences from the environment corrupt the heat flow from the sample to the IR camera, Fig. 2., column 1. Raw data were processed with the Fast Fourier Transformation algorithm. The amplitude image and phase image taken at a frequency of $0.1953 \mathrm{~Hz}$ were selected from the result set. The amplitude image (Fig. 2., column 2) shows a great impact of uneven heating, while phase images (Fig. 2., column 3) have proven to be quite successful in finding simulated damages. The last column shows the results after Principal Component Analysis. The results given is the Empirical Orthogonal Function $\left(E O F_{2}\right)$. Images clearly show the edges of the damaged area, but results are still contaminated by the effects of uneven heating.

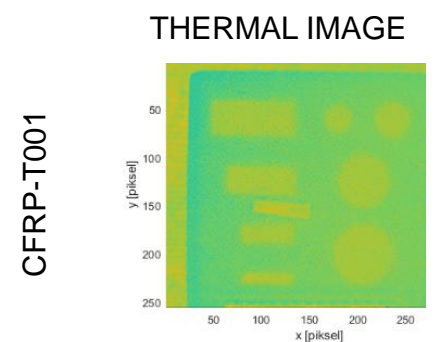

AMPLITUDE IMAGE
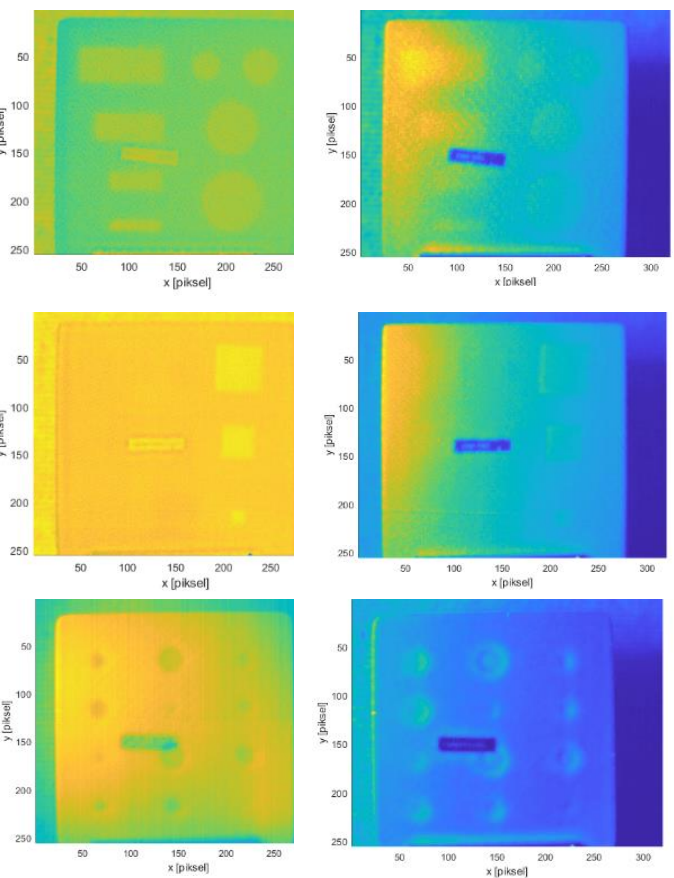

PHASE IMAGE
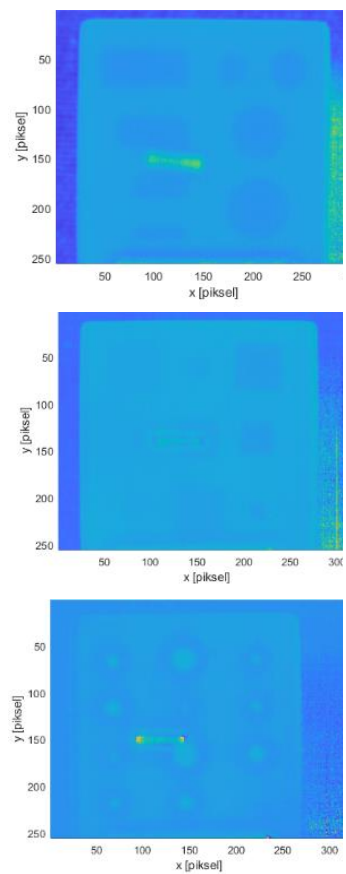

PCA
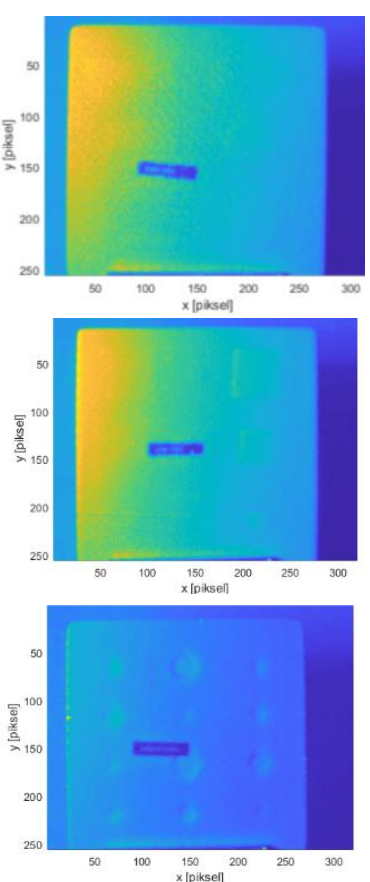

Fig. 2. Samples CFRP-T001, CFRP-T002 and SENDWICH001: raw image and after processing with PPT (amplitude and phase image) and PCA method.

\section{REFERENCES}

[1] R. E. Martin, A. L. Gyekenyesi, S. M. Shepard, Interpreting the Results of Pulsed Thermography Data. Materials Evaluation, Vol. 61, no. 5, pp. 611-616, 2003.

[2] X. Maldague, S. Marinetti. Pulse Phase Infrared Thermography. J. Appl. Phys, 79:2694-2698, 1996.

[3] N. Rajic. Principal component thermography for flaw contrast enhancement and flaw depth characterisation in composite structures. Composite Structures 58 (2002) 521-528, 2002. 